\title{
Líquen plano oral bolhoso: relato de experiência
}

\author{
Oral bullous lichen planus: case detailing
}

Liquen plan oral bulloso: relato de experiencia

Catarina Brito da Rocha Medeiros ${ }^{1}$, Beatriz Vieira Nunes ${ }^{1}$, Andréa Tatiane Oliveira da Silva ${ }^{1}$, Aurea Valéria de Melo Franco ${ }^{1}$, Fernanda Braga Peixoto ${ }^{1}$, Vanessa de Carla Batista dos Santos $^{1 *}$, Sonia Maria Soares Ferreira ${ }^{1}$.

\section{RESUMO}

Objetivo: Relatar um caso de Líquen Plano Oral Bolhoso, expondo características clínicas e histopatológicas da lesão. Relato de experiência: Paciente sexo feminino, 86 anos, compareceu ao serviço, queixando-se de "saliência" em palato duro. Ao exame intraoral, foi detectado, placa vermelha, lisa, irregular e sintomática. A hipótese diagnostica foi de eritroplasia. Realizada biópsia incisional e microscopicamente observados fragmentos de mucosa revestidos por epitélio estratificado pavimentoso ceratinizado atrófico com áreas de epidermização, hiperceratose, exocitose e degeneração hidrópica da camada basal e a junção epitélio conjuntivo apresentava-se com bolha subepitelial e área focal de projeção em dentes. Após ser diagnosticada a paciente foi submetida à terapia medicamentosa e encontra-se em acompanhamento. Considerações finais: O conhecimento dessa patologia é de fundamental importância, para o diagnóstico precoce e tratamento adequado, visto que é uma lesão rara e de difícil diagnóstico, porém com bom prognóstico.

Plavras-chave: Líquen plano bucal, Cavidade oral, Patologia.

\begin{abstract}
Objective: Is to report an Oral Bullous Lichen Planus case, exposing clinical and histopathological characteristics of the injury. Case detailing: Patient female, 86 years old, attended the service, complaining about a "protrusion" on the hard palate. On the intraoral examination, a smooth, irregular and symptomatic red plaque was detected. The hypothesis diagnosed was erythroplakia. An incisional and microscopically observed mucosal fragments were coated with stratified atypical keratinized squamous epithelium with areas of epidermis, hyperkeratosis, exocytosis and hydropic degeneration of the basal layer and the conjunctive epithelial junction presented with subepithelial bubble and focal area of projection in teeth. After being diagnosed, the patient was submeter to drug therapy and is being followed up. Final considerations: The knowledge of this pathology is of fundamental importance, for the early diagnosis and appropriate treatment, whereas it is a rare injury and difficult diagnostic.
\end{abstract}

Key words: Lichen buccal plane, Oral cavity, Pathology.

\section{RESUMEN}

Objetivo: Es informar sobre un caso de Liquen Plan Oral Bulloso, presentando características clínicas e histopatológico de la lesión. Relato de experiencia: Paciente sexo femenino, 86 años, llegó al ambulatorio quejándose de un abultamiento en el palato duro. Al practicarle un exámen intra-oral, se encontró una mancha roja, lisa, irregular y sintomática. La hipótesis diagnosticada fue de eritroplasia. Al realizarle biopsia incisional y microscópicamente observados los fragmentos de mucosas forrados por

${ }^{1}$ Centro Universitário CESMAC, Maceió, Alagoas, Brasil. *E-mail: nessadecarlla@yahoo.com.br 
epitélio estratificado escamoso, queratinizado atrófico con áreas de epidermización hiperqueratosis exocitosis degeneración hidrópica de la capa basal y la unión epitelial conjuntiva se presentaba con burbuja subepitelial y área focal de proyección en dientes. Después de ser diagnosticada la paciente fue sometida a terapia medicada con acompañamiento. Consideraciones: El conocimiento de esta patología es de fundamental importancia, para el diagnóstico precoz y el tratamiento adecuado, ya que es una lesión rara y difícil de diagnosticar, sin embargo con buen pronóstico.

Palabras clave: Liquen plan oral, Cavidad oral, Patologia.

\section{INTRODUÇÃO}

O líquen plano oral (LPO) é uma patologia de origem desconhecida, mucocutânea crônica de natureza inflamatória e autoimune, nela há um mecanismo imunopatológico onde verifica-se a agregação linfocitária do tipo T a frente das células basais do epitélio da cavidade oral (FARIAS BS, et al., 2014).

Diversas causas foram relacionadas, como: diabetes, doenças autoimunes, doenças intestinais, drogas, hipertensão, infecções, materiais dentários, neoplasias, predisposição genética. (ISMAIL SB, et al., 2007; SCULLY C, et al., 2008).

Histopatologicamente o assalto linfocitário localizado aos queratinócitos da camada basal da mucosa é um dos fatores principais do líquen plano oral (ISMAIL SB, et al., 2007).

A apoptose e a degeneração celular são ocasionadas pelos linfócitos T e os mesmos perduram o método ao liberar quimiocinas na inflamação (RIBEIRO S, et al., 2015).

Para Cervantes GS, et al., (2008), as condições psicológicas, em especial salientes graus de estresse e ansiedade, estão diretamente associados ao líquen plano. Costumeiramente, essa disfunção ocorre na mucosa oral, porém verfica-se que é capaz de aparecer na pele, unhas e genitais.

Habitualmente afeta mais mulheres que homens e o envolvimento de crianças é raro (NEVILLE, B.W, et al., 2016; RIBEIRO S, et al., 2015). Para Fraga HF, et al., (2011), indivíduos de raça branca possuem um risco cinco vezes maior de desenvolver a doença, comparado as demais raças.

O diagnóstico dessa patologia deve ser feito através de exames clínicos e histopatológicos, há determinantes lesões onde apenas o olhar clínico já consegue diagnosticar o líquen, porém para comprovar o diagnóstico, eliminar níveis de displasia e malignidade, é necessária a biópsia. (FARIAS BS, et al., 2014).

Para Cervantes GS, et al., (2008), o líquen plano bucal possui características distintas e fáceis de serem identificadas, dispõe de seis apresentações clínicas representadas na literatura como: reticular, papular, em placa, erosiva, atrófica e bolhosa.

Para Martins R, et al., (2008), a principal característica do líquen plano reticular é a presença de finas linhas brancas intituladas de "estrias de Wickham", que se unem em um padrão anular ou rendilhado. Comumente essas lesões não são estáticas, podendo melhorar e piorar em semanas ou meses. As lesões, geralmente, são assintomáticas, bilateral, simétrica e afetam a mucosa jugal posterior em grande parte das situações. (CANTO AM, et al., 2010).

O líquen plano oral papular é dificilmente observado e comumente vem acompanhado por outra nuance. Ele demonstra pequenas pápulas brancas $(0,5 \mathrm{~mm}$ a $1 \mathrm{~mm}$ de diâmetro) com estrias finas em seu contorno (FARIAS BS, et al., 2014).

Segundo Fraga HF, et al., (2011) o tipo placa demonstra o encontro de pápulas e estrias que formam placas brancas com distribuição multifocal, onde podem ser levemente elevadas ou quase planas, atingindo principalmente a mucosa jugal e o dorso de lingua. Frequentemente não apresenta sintomas e não requer tratamento específico. 
Para Canto AM, et al., (2010) a forma clínica mais significativa é a erosiva, pois consiste em lesões sintomáticas e são associadas a possibilidade de transformação maligna. A forma atrófica apresenta-se como uma área rosada, eritematosa, pertinente ao afinamento do epitélio expondo os pequenos vasos sanguíneos das áreas inflamadas. Assim como na eritroplasia apresenta áreas eritematosas e inflamatórias, sendo assim necessário seu diagnóstico diferencial (CARRION B, et al., 2008).

O tipo bolhoso é a forma clínica mais atípica, apresentando bolhas que aumentam de tamanho e tendem à ruptura, deixando a superfície ulcerada e dolorosa. A margem da lesão é, em geral, cercada por estrias finas e queratinizada. Acometendo com maior frequência mucosa jugal em região de segundos e terceiros molares e borda lateral de lingua (CANTO AM, et al., 2010). A histopatologia caracteriza-se por bolhas subepiteliais, acantose, degeneração hidrópica da camada basal e denso infiltrado linfocitário (RIBEIRO S, et al., 2004).

O tratamento visa uma melhor qualidade de vida, buscando aliviar sintomas, dado que a cura nem sempre é possível (FRAGA HF, et al., 2011).

Para Lapiedra C, et al., (2008), o tratamento é sintomático e baseia-se principalmente no uso de corticoides, tópicos e sistêmicos. Uso de corticoides apresenta boa efetividade, e alguns pacientes apresentam remissão completa dos sintomas por determinado período de tempo (FARIAS BS, et al., 2014).

O objetivo do presente estudo é relatar as características clínicas e histopatológicas do Líquen Plano Oral Bolhoso, um subtipo raro de Líquen Plano Oral.

\section{RELATO DE CASO}

Paciente, melanoderma, 86 anos, sexo feminino, compareceu ao serviço de Odontologia de uma clínica escola, queixando-se de irritação e saliência no palato há cerca de 20 anos. A paciente é portadora de doenças sistêmicas como diabetes melitus, hipercolesterolemia, hipertensão arterial sistêmica (HAS), labirintite e faz o uso das medicações como losartana, sivastatina, metiformina e alodipina. Ex-fumante, a mesma fumou por 55 anos e parou há 20 anos, etilista há trinta anos. Ao exame extrabucal nenhuma alteração foi observada.

Ao exame intrabucal observou-se uma lesão do tipo erosão em um leito com manchas hipercrômicas e leucoplásicas, envolvendo o terço médio do palato duro, sintomatologia dolorosa tipo ardência ao ingerir alimentos quentes (Figura 1 A e B). As principais hipóteses diagnósticas foram eritroplasia, leucoeritroplasia, líquen plano erosivo e carcinoma de células escamosas.

Figura 1 (A e B) - Lesão do tipo erosão, circundada por manchas hipercrômicas e leucoplásicas, localizada em palato duro.
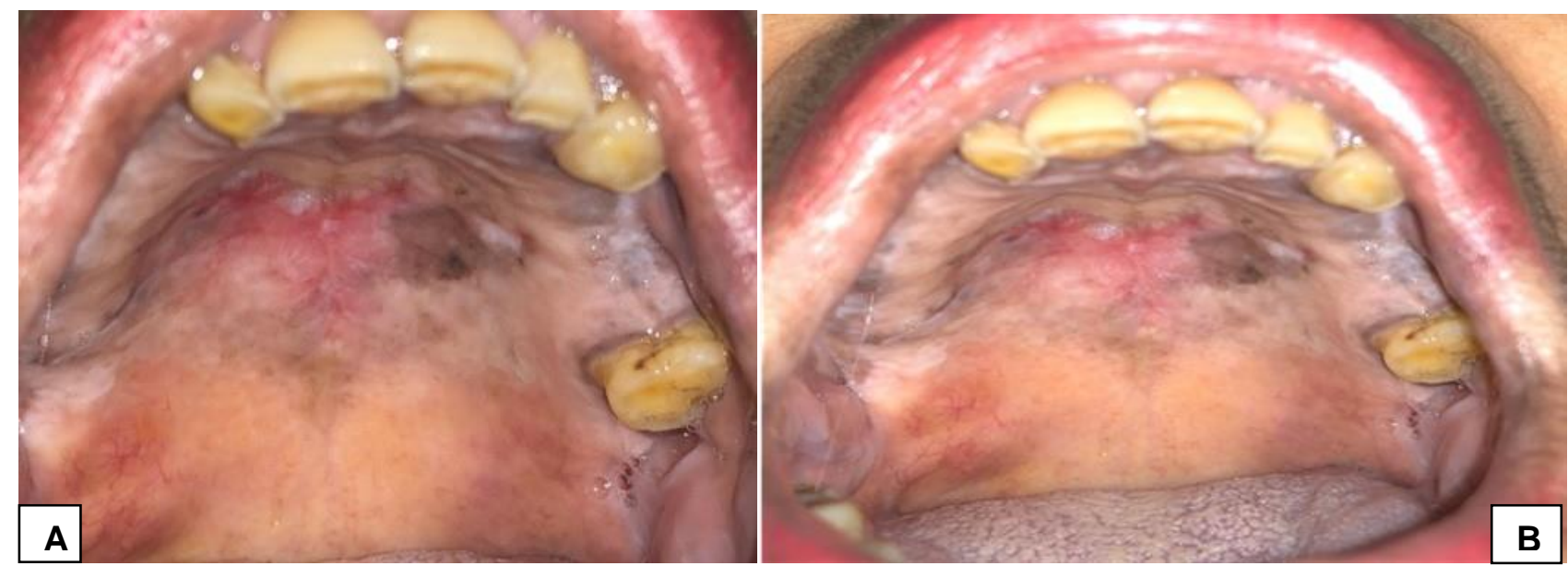

Fonte: Medeiros CBR, Nunes BV, Silva ATO, et al., 2017. 
Foi realizada uma biópsia incisional e a amostra foi encaminhada ao Serviço de Patologia para análise histopatológica. Os cortes histológicos revelaram fragmentos de mucosa revestidos por epitélio estratificado pavimentoso ceratinizado atrófico de epidermização, hiperceratose, exocitose e degeneração hidrópica da camada basal. A junção epitélio conjuntivo apresentava-se com bolha subepitelial e área focal de projeção em dentes de serra. A lâmina própria era fibrosa e apresentava intenso infiltrado inflamatório subepitelial e disposto em faixa com áreas discretas de incontinência pigmentar. Fragmentos de glândulas salivares menores hialinizados com infiltrado inflamatório linfocitário periglandular estão presentes complementando o quadro microscópico (Figura 2 e 3).

Figura 2 - Fotomicrografia da lesão bucal, na seta azul observa-se que a junção epitélio conjuntivo apresentava-se com bolha subepitelial. Imagem no aumento de 40x, coloração: Hematoxilina-Eosina (HE).

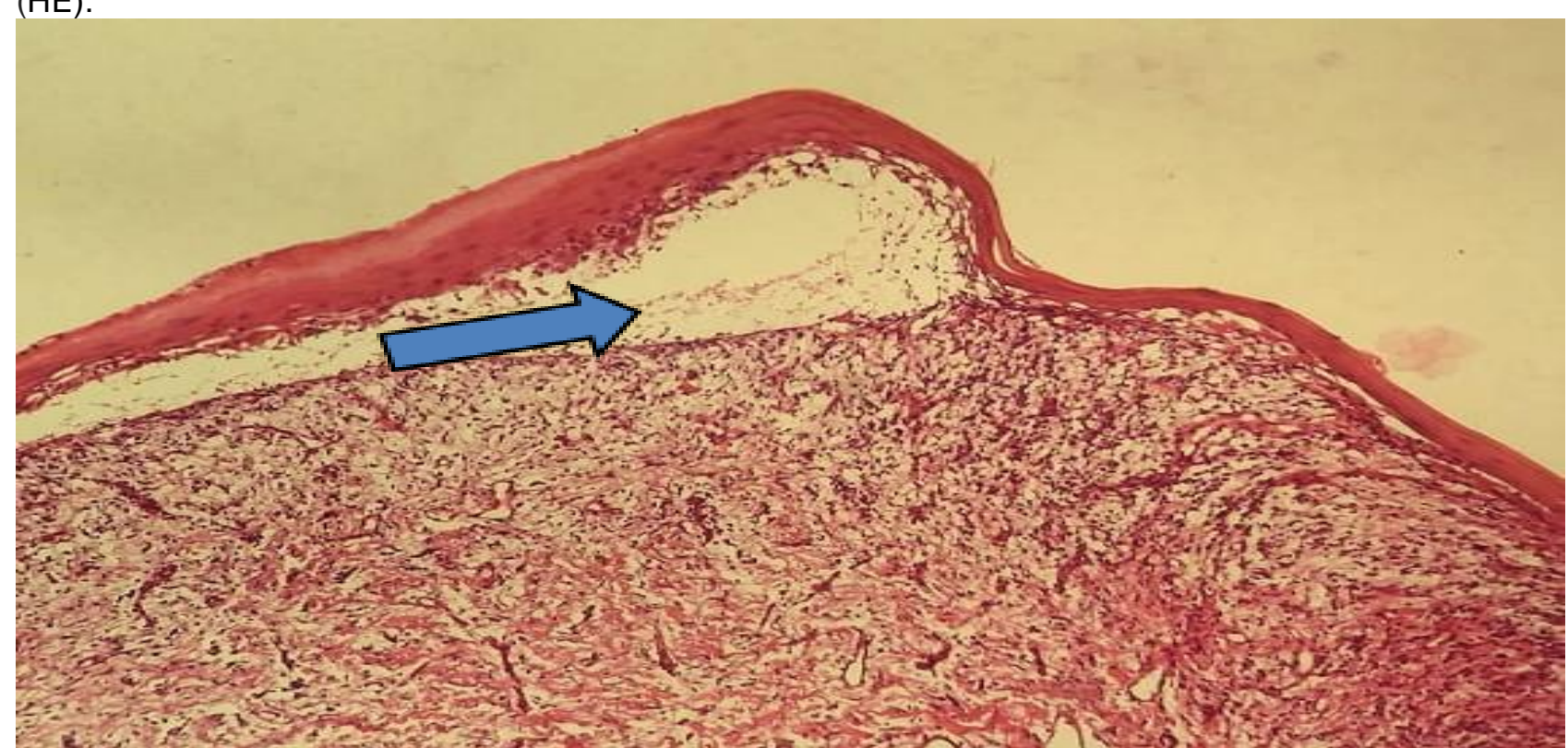

Fonte: Medeiros CBR, Nunes BV, Silva ATO, et al ., 2017.

Figura 3 - Fotomicrografia da lesão bucal, a seta azul exibe epitélio estratificado pavimentoso ceratinizado atrófico. Imagem no aumento de 100x, coloração: Hematoxilina-Eosina (HE).

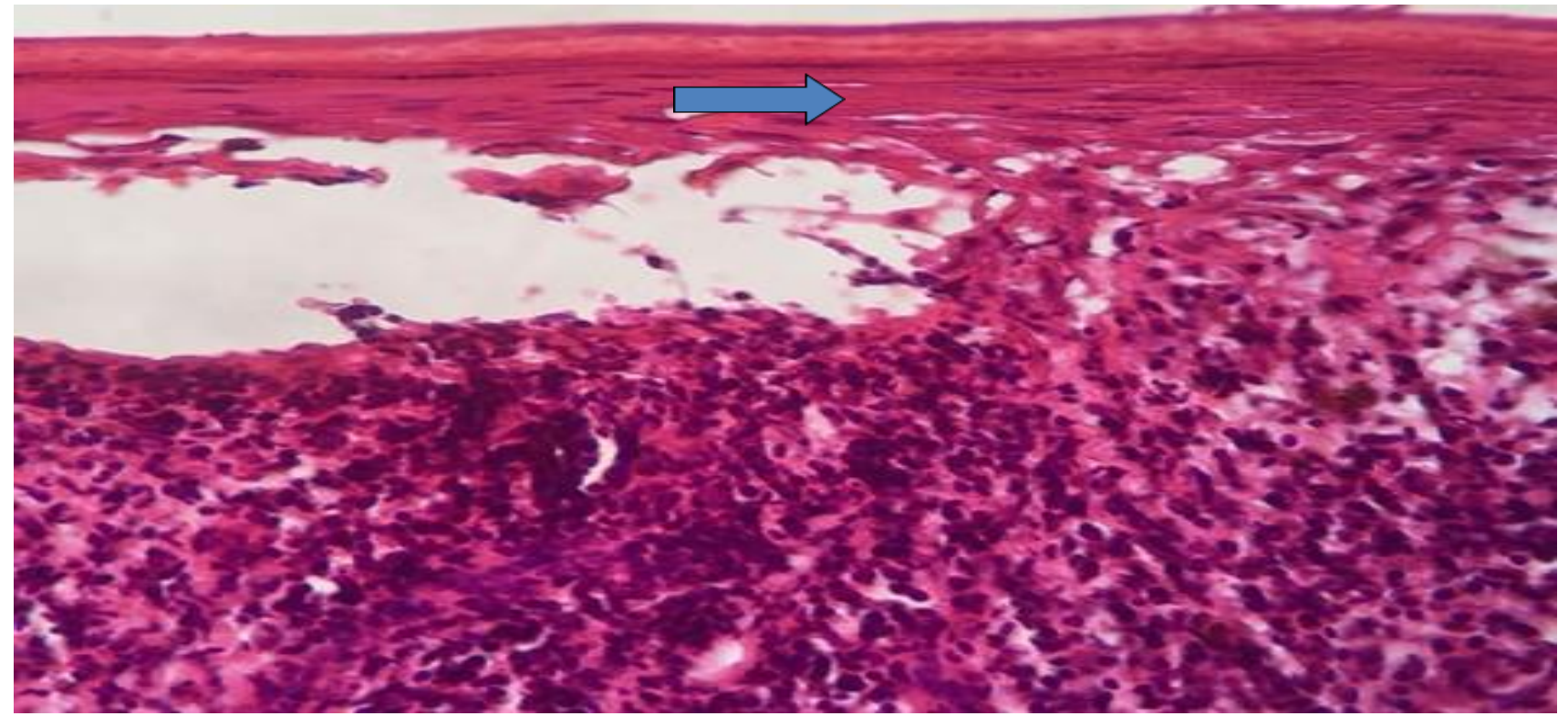

Fonte: Medeiros CBR, Nunes BV, Silva ATO, et al ., 2017. 
A paciente foi medicada inicialmente com CLOB-X GEL (corticoide tópico) na região e em seguida foi sugerido trocar a medicação por extrato fluido de Chamomilla recutita (L.) Rauschert (AD-MUC). Os sintomas desapareceram e as lesões regrediram totalmente.

\section{DISCUSSÃO}

Segundo Ribeiro S et al. (2004) o LPO bolhoso é a forma mais incomum de líquen plano oral. É descrito como pequenas bolhas ou vesículas que se rompem espontaneamente, quando isso acontece, há ulceração na superfície com sintomatologia (RIBEIRO S, et al., 2004).

Esses achados corroboram com os encontrados no presente estudo, que foi observado apenas uma área erosada sem formação de bolhas. Porém difere dos achados na literatura quando diz respeito a sintomatologia, pois a paciente relatou sintomatologia dolorosa, durante a ingestão de alimentos quentes.

De acordo com a localização mais prevalente, alguns estudos (GONÇALVES LM et al., 2018; CANTO AM, et al., 2010), relatam que os principais sítios são em mucosa jugal, lábios e dorso da língua. No referido caso a paciente apresentava lesão no terço médio do palato duro, sendo considerada uma área incomum de ser acometida.

Estudos apontam que o LPO é uma patologia crônica de natureza inflamatória e auto-imune, onde afeta adultos de meia-idade, há preferência por mulheres em uma proporção de 3:2 (CANTO AM, et al., 2010; FARIA BS, et al., 2014). O caso relatado corrobora com os dados da literatura referentes à predileção do líquen plano oral por mulheres. Por outro lado a faixa etária a qual a paciente pertence não se encaixa com os dados encontrados na literatura.

Estudos afirmam que a histopatologia do LPO consiste em degeneração hidrópica da camada basal, intenso infiltrado inflamatório e hiperqueratose (CANTO AM, et al., 2010). O referido caso vai de acordo com a literatura, apresentando bolha subepetielial que é característica principal para o diagnóstico da lesão, degeneração hidrópica da camada basal, hiperceratose e intenso infiltrado inflamatório subepitelial.

O tratamento do LPO tem como objetivo a melhoria da qualidade de vida, aliviando os sintomas e a inflamação, dado que a cura nem sempre é possível (FARIA BS, et al., 2014). O uso de corticoides apresenta boa efetividade, e alguns pacientes apresentam remissão completa dos sintomas por determinado período de tempo (FARIA BS, et al., 2014).

No caso apresentado, a paciente foi medicada inicialmente com CLOB-X GEL (corticoide tópico) na região e em seguida foi sugerido trocar a medicação por AD MUC. Os sintomas desapareceram e as lesões regrediram totalmente. O conhecimento dessa patologia pelo cirurgião-dentista é de fundamental importância, cabendo a esse profissional uma parcela de responsabilidade pelo diagnóstico precoce e pela orientação para o tratamento adequado.

\section{CONSIDERAÇÕES FINAIS}

O Líquen Bolhoso é uma lesão rara incomum em cavidade oral, porém, quando ocorrem, as lesões são bastante sintomáticas ocasionando incômodo ao paciente. O conhecimento dessa patologia é de fundamental importância, para o diagnóstico precoce e tratamento adequado, visto que é uma lesão rara e de difícil diagnóstico, porém com bom prognóstico.

\section{REFERÊNCIAS}

1. CANTO AM, et al. Líquen plano oral (LPO): diagnóstico clínico e complementar. An. Bras. Dermatol, 2010; 85(5); 669-675.

2. CARRIÓN B, et al. Diagnóstico del liquen plano oral. Av. Odontoestomatol, 2008; 24 (1): 11-31.

3. CERVANTES GS, et al. Líquen plano bucal: considerações clínicas e histopatológicas. Brazilian Journal of Otorhinolaryngology, 2008; 74(2):284-292. 
4. FARIA BS, et al. Líquen Plano Oral em Lábio Inferior: Relato de Caso. Revista brasileira de ciências da saúde, 2014; 18(1):55-62.

5. FRAGA HF, et al. A importância do diagnóstico do líquen plano bucal. J Health Sci Inst. 2011; 29(1):27-30.

6. GONCALVES LM, et al. Avaliação clínica das lesões orais associadas a doenças dermatológicas. An. Bras. Dermatol, 2010, 85(2):150-156.

7. ISMAIL SB, et al. Oral lichen planus and lichenoid reactions: etiopathogenesis, management and malignant transformation. J Oral Sci 2007; 49:89-106.

8. LAPIEDRA C. Malignización del liquen plano oral. Av Odontoestomatol, 2008; 24(1); 97-103.

9. MARTINS R, et al. Líquen plano bucal. RSBO Revista Sul-Brasileira de Odontologia, 2008; 5(2):64-68.

10. NEVILLE BW, et al. Patologia Oral e Maxilofacial. Trad.4a Ed., Rio de Janeiro: Elsevier, 2016.

11. RIBEIRO S, et al. Líquen plano bolhoso oral: relato de caso clínico. UFES Ver odontol, 2004; 6(3):61-66.

12. SCULLY C, CARROZZO M. Oral mucosal disease: lichen planus. Br J Oral Maxilofac Surg. 2008; 46:15-21. 